\title{
The Exploration of Biasing Voltage Range on Implantable Microscale Electrochemical Sensors for Post-Surgery Cancer Recurrence Monitoring
}

\author{
Lidi Jie \\ Scottish Microelectronics Centre, the University of Edinburgh, Edinburgh City, UK
}

Email address:

1loydjie@ed-alumni.net

\section{To cite this article:}

Lidi Jie. The Exploration of Biasing Voltage Range on Implantable Microscale Electrochemical Sensors for Post-Surgery Cancer Recurrence Monitoring. American Journal of Chemical Engineering. Vol. 3, No. 6, 2015, pp. 74-79. doi: 10.11648/j.ajche.20150306.11

\begin{abstract}
Cancer surgery prognosis currently relies heavily on expensive diagnostics devices that have limited availability to patients. Minute-to-minute cancer recurrence monitoring device is needed. This paper investigates a novel design and simulation of Implantable micro-scale, minute-to-minute sensors of low manufacture cost. In Cadence simulation software, electrochemical sensor array circuit in 0.35 micron complementary metal oxide semiconductor (CMOS) process was designed. The simulated circuit supported both pH ISFET sensor and soluble oxygen level O2-FET sensor. The simulation result showed that the circuit's output voltage has optimal sensitivity when ISFET $V_{\text {gate }}$ was biased at range of 2.29-2.49 volts. The output voltage in this biasing scheme varies linearly with the input voltage $\mathrm{V}_{\text {gate }}$ making it ideal region for monitoring of cancer. The appropriate biasing of ISFET $\mathrm{V}_{\text {gate }}$ voltage found by simulation is within 2.29-2.49 volts. In the case of $\mathrm{pH}$ measurements, the sensitivity can be up to $0.02 \mathrm{pH}$ in a minute-to-minute $\mathrm{pH}$ probing device for cancer prognosis tracking. Other chemical probes such as O2-FET and Oct4 sensors are also supported by the device architecture.
\end{abstract}

Keywords: Bioelectronics, Electrochemical Sensor, Cancer Prognosis, Microelectromechamical Systems (MEMS), pH, Nanowire-Sensor

\section{Introduction}

Cancer surgery prognosis has long been an issue as the recurrence of cancer occurs after the operation. The survivorship rates for prevalent cancer types are considerably low. The 2014 data showed post-treatment survival rate is only $9 \%$ in American male colorectal cancer patients and $8 \%$ in American female uterine colon and rectum cancer patients [1]. Additionally due to the lack of the availabilities of the early-stage tumor diagnostics devices apparatuses such as PET (positron emission tomography), many of such patients in developing countries do not get cancer redevelopment diagnostics and the cancer can resume unnoticed after surgery. To solve this problem, our paper introduces a novel cheap implantable and biocompatible electrochemical sensor for cancer surgery prognosis. Such that doctors can implant the post-surgery cancer monitoring device into the patient's cancer-affected organ and get minute-to-minute monitoring of any cancer cells growing back. The electrochemical sensing technology involved is a popular field of cross-disciplinary research with industrial applications in a variety of markets. The main advantage of MEMS (microelectromechamical systems) electrochemical sensing technology to some microscopy-coupled biophysical MEMS sensors is that the electrochemical sensor does not need external facilities such as atomic force microscopy in analyses of the measurements as in silicon MEMS resonators [2]. Thus, the patients can just monitor the cancer recurrence at home with the wireless sensor reader, once the wireless-communication coupled electrochemical sensor is implanted, without having to go to hospitals.

Previous research at Glasgow University has demonstrated that array sensor circuits can be used in minute-to-minute monitoring of $\mathrm{pH}$ for slime molds [3]. The advantages of the sensor array circuit is that it allows for the continuous sampling of the slime molds and the results obtained of the arrays can be synthesized to produce a camera-like image. The sensor array research of Glasgow group however can be further extended to monitor the exact pixel location of the cancer recurrence after the surgery has taken place. The complementary metal oxide semiconductor (CMOS) 
compatible device can be manufactured at cheap scale. The minute-to-minute monitoring of cancer recurrence may also possess advantages such as cheapness and convenience over the current PET (Positron Emission Tomography) or CT (computed-tomography) in that the PET costs up to $1000 \mathrm{US}$ dollars per trial and take months to schedule for a diagnostic appointment. While our newly proposed sensor array device can provide continuous $24 / 7$ monitoring of cancer recurrence upon surgery implants.

The array circuit architecture supports various types of sensors to be loaded on to the probes positions of individual array cells. The circuit can cater the needs for $\mathrm{pH}$ and dissolved oxygen concentration measurements. The possible probes attached to the circuit array architecture include the ISFET (Ion Sensitive Field Effect Transistor) which allow for pH measurements and O2-FET (oxygen FET) which allow for dissolved oxygen measurements.

The electrochemical-FET sensors were designed in Cadence simulation software in AMS $0.35 \mu \mathrm{m}$ CMOS technology before the assembly and testing of the circuits in the lab of Scottish Microelectronics Centre. Next step of research includes incorporation of wireless digital circuits for the transmission of the implanted device's measurements. The final product needs also address the issue of implantable biocompatibility, such that the implanted device will not cause harm to the human tissues and organs. A proposed solution is to deposit a layer of parylene encapsulation on top of the MEMS device in the device packaging. The parylene is an American Food and Drug Administration (FDA) approved material that has been used in medical sensor device implants [4].

\section{Methods}

Software simulation by Cadence was used to find the effective region of operation for the $\mathrm{V}_{\text {gate }}$ of the ISFET. The simulation can also be used for oxygen level concentration and a separate equation should be formulated for converting oxygen concentration to output voltage at $\mathrm{V}_{\text {drain }}$ of the individual cells in the array. In our research of electrochemical sensor for cancer detection, the solution being tested is the tissue's extracellular fluid and replaces the gate dielectric as the ion sensitive membrane. This novel ISFET design differs from the traditional Bergveld's ISFET where the gate dielectric or $\mathrm{SiO}_{2}$ serves as the ion sensitive membrane. With the extracellular fluid replacing gate dielectric the surface potential is controlled by the $\mathrm{pH}$ of the solution [5]. The ISFET functions similar to a MOSFET in that the drain current $I_{d}$ is a function of gate-source voltage $V_{G S}$ as in (1).

$$
\mathrm{Id}=\mu \operatorname{Cox} \frac{\mathrm{W}}{\mathrm{L}}\left(\left(V_{G S}-\left|V_{T}\right|\right) V_{D S}-\left(\frac{V_{D S}}{2}\right)^{2}\right)\left(1+\lambda V_{D S}\right)
$$

Note that (1) illustrates the correlation Drain current expression in linear regime as a function of the ISFET gate-source voltage.

As the ion-sensitive membrane serving as the gate of the ISFET, the $\mathrm{pH}$ variance of the tissue environment being tested can affect the surface potential at the gate of the ISFET as in (1).

$$
\frac{\delta \psi_{0}}{\delta \mathrm{pH}_{\mathrm{B}}}=-2.3 \frac{\mathrm{kT}}{\mathrm{q}} \alpha
$$

As (2) illustrates the surface potential at the oxide solution interface varies linearly with the $\mathrm{pH}$ value of the bulk solution. $\mathrm{k}$ is the Boltzmann constant, $\mathrm{T}$ is the absolute temperature and $\mathrm{q}$ is the charge of an electron [5]. The coefficient $\alpha$ is defined as a parameter in between 0 and 1 .

In the Scottish Microelectronics Centre's Austria Microsystem (AMS) 0.35 micron technology simulation environments we have built the O2-FET compatible sensor array circuit adapted from the design of Prof. Cumming's group at the University of Glasgow as shown in Fig. 1 [6].

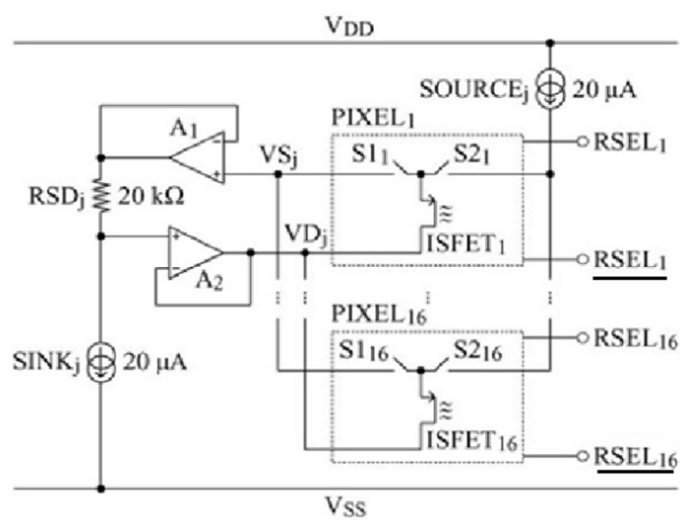

Figure 1. Schematics of a column of 16 pixels in the sensor array circuit designed by Cumming's group at The University of Glasgow.

As illustrated on Fig.1, with $\mathrm{V}_{\mathrm{ds}}$ constant at $20 \mathrm{k} \Omega * 20 \mu \mathrm{A}=$ $0.4 \mathrm{~V} . \mathrm{V}_{\mathrm{GS}}-\mathrm{V}_{\mathrm{T}}$ has to stay constant to keep drain current $\mathrm{I}_{\mathrm{d}}$ constant at $20 \mu \mathrm{A}$. With $\mathrm{V}_{\mathrm{T}}$ also kept constant, $\mathrm{V}_{\mathrm{d}}$ or $\mathrm{V}_{\mathrm{s}}$ changes will reflect the $\mathrm{V}_{\text {gate }}$ or the $\mathrm{pH}$ change.

All components of the circuit including resistors, op-amps, switches and current sources are built from transistors and can be manufactured by Austria Microsystem (AMS) 0.35 micron process. Since the scale of the transistors is of 0.35 micron process and is much smaller than typical circuit components such as resistors, op-amps, switches and current sources. This design enables the sensor device to be embedded in an implantable microscale chip.

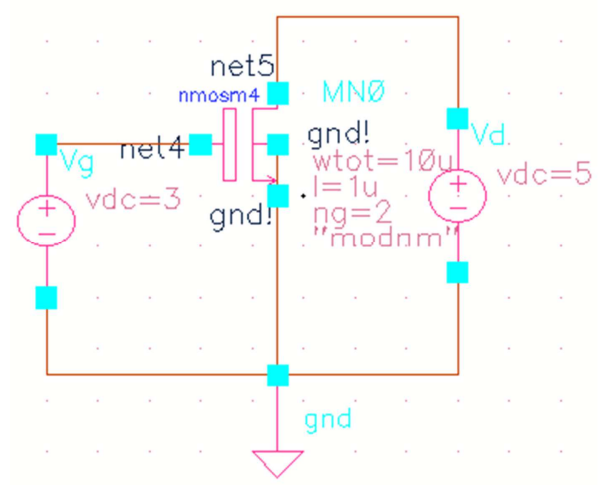

Figure 2. NMOS transistor functioning as a resistor simulated in Cadence software. 
The transistor-resistor would give 20 kilo ohms resistance. The transistor is in linear mode of operation and thus functions like a resistor as the ratio of voltage across the transistor over the current through it is a constant. The simulation plot of Fig. 2 is shown in figure and by ohm's law we can confirm the equivalence resistance of the simulated transistor-based device is 20 kilo-ohms.

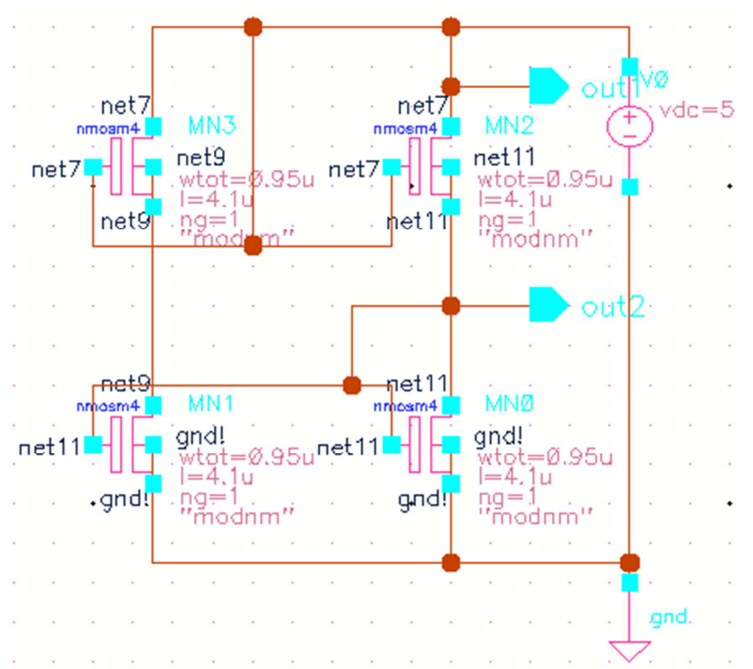

Figure 3. Transistors functioning as a current source simulated in Cadence software.

As shown in Fig. 3, the current source would produce 20 micro-amperes current. CMOS-designed $20 \mu \mathrm{m}$ current source or sink circuit schematics, the output is in MN2, top right transistor, with out 1 connected the transistor drain and out 2 connected to transistor source.

The ISFET is being simulated by width $10 \mu \mathrm{m}$ and length $0.5 \mu \mathrm{m}$ is coupled with drain, source capacitor, gate and bulk to make a PMOS-ISFET. The simulation schematics show the actual circuit components details views in the layout hierarchy of Cadence Simulation Software. The actual circuitry embedded in the lower level while in the top level view in Cadence. The PMOS-ISFET is just shown as a block diagram in Fig. 4.

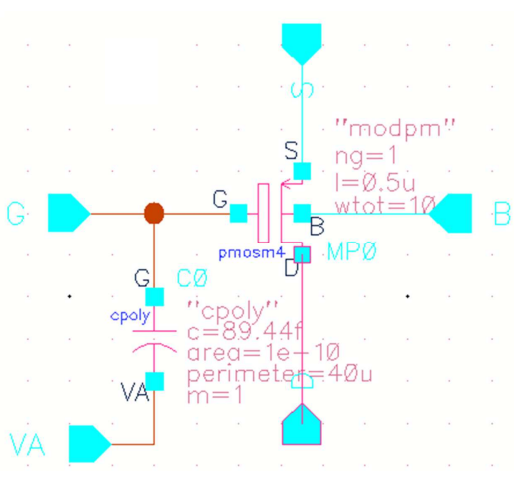

Figure 4. PMOS-ISFET of the sensor array circuit, simulated in Cadence software.

The top node is the source and the bottom node is the drain. A capacitor is being coupled at the gate node with capacitance 89.44 farad.

Following the standard procedures of hierarchical circuit components and entire circuitry, only after each individual component was simulated and tested, the entire circuit was assembled together. The individual circuit components simulations were selectively shown in Fig. 5 (the resistor constructed of transistors) and Fig. 6 (the current source or sink constructed of transistors).The device of the entire simulation circuit including components of resistors, current sources and switches etc. was simulated in a plot as shown in Fig. 7 of the result section.

All parameters in the circuit simulation design were tested and were subjected to modifications till optimal results were obtained. After all components of the circuit were tested the circuit was assembled together.

\section{Results}

\subsection{Components'Simulation Results}

The current source or sink is shown in Fig. 3. All four transistors are NMOS of length $4.1 \mu \mathrm{m}$ and width $0.95 \mu \mathrm{m}$. The current source outputs $19.95 \mu \mathrm{A}$ current. The simulated result is shown in Fig. 5.

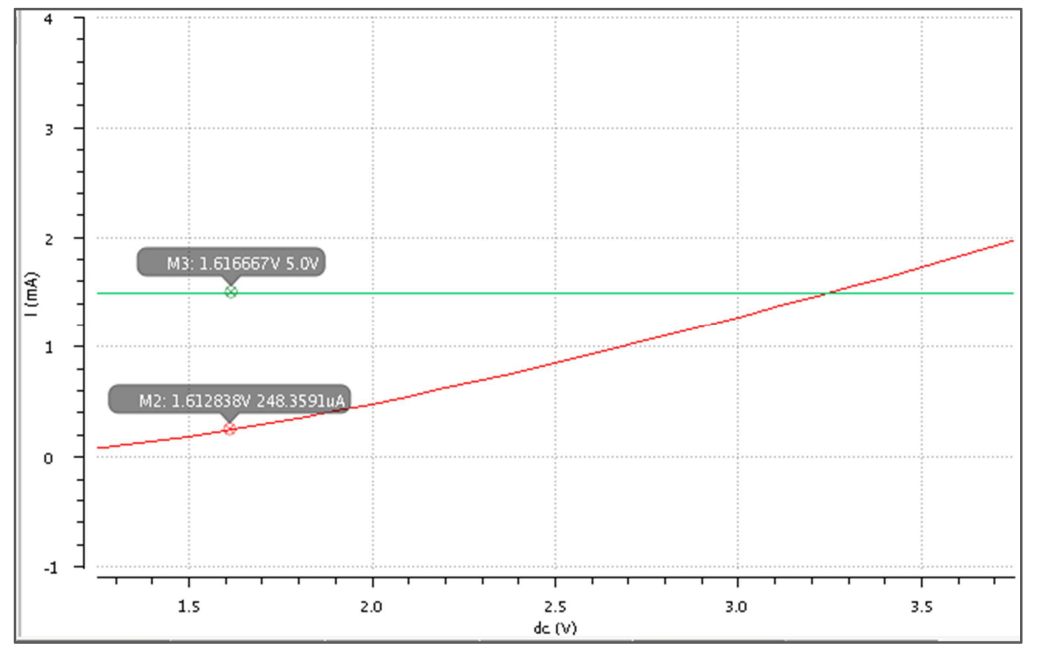

Figure 5. The plots of voltage and current versus gate voltage sweep. The voltage of the drain (constant at $5.0 \mathrm{~V}$ ) and the rising current across the resistor. 


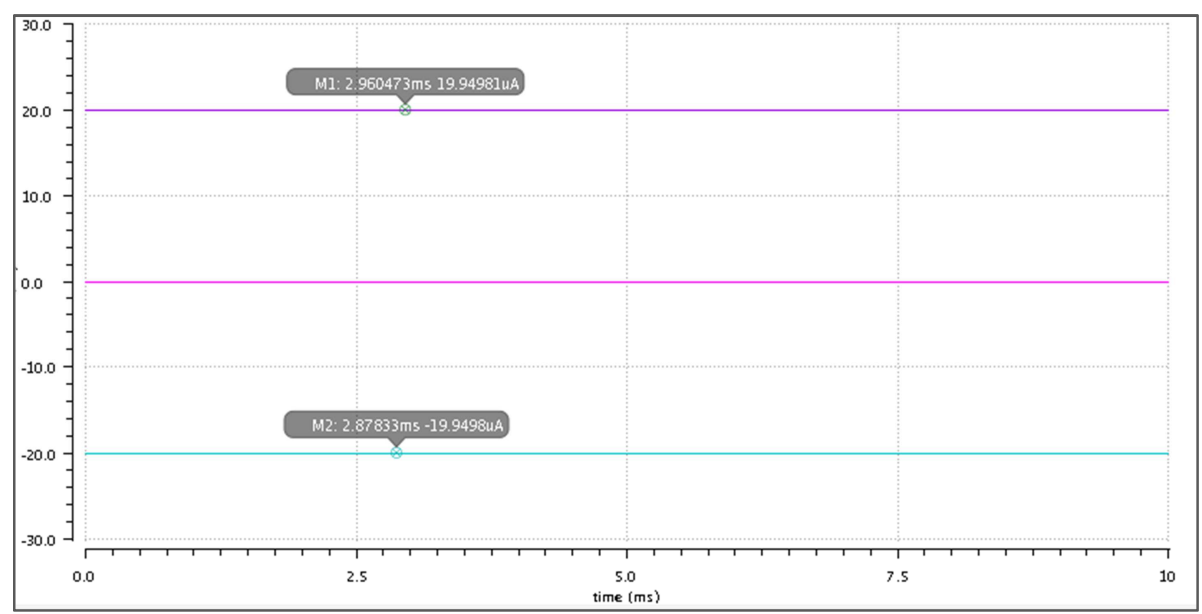

Figure 6. Simulation results of current source or sink circuit, with the transistor M2 drain current output as positive and source current output negative.

\subsection{Sensor Circuit Simulation Results}

The overall simulation result is shown in Fig. 7. In the complete sensor array (single row) circuit as shown in Fig. 7, we swept the gate voltage $\mathrm{V}_{\text {gate }}$ of the PMOS-ISFET and the results were measured of the voltages of the drain and the source of the PMOS-ISFET. The threshold voltage of turning on the PMOS-ISFET is around 1.7 volts where the $\mathrm{V}_{\text {drain }}$ and
$\mathrm{V}_{\text {source }}$ cease from being constant and start to rise in gradient $=1$ linear curves upward.

To find the region of optimal operation, gate voltage of ISFET $\mathrm{V}_{\text {gate }}$ was sweeped from $0 \mathrm{~V}$ to $5 \mathrm{~V}$ while keeping the circuit's voltage within the conventional $5 \mathrm{~V}$ supply for the chip power supplies. The result of the drain and source voltages are displayed in Fig. 5.

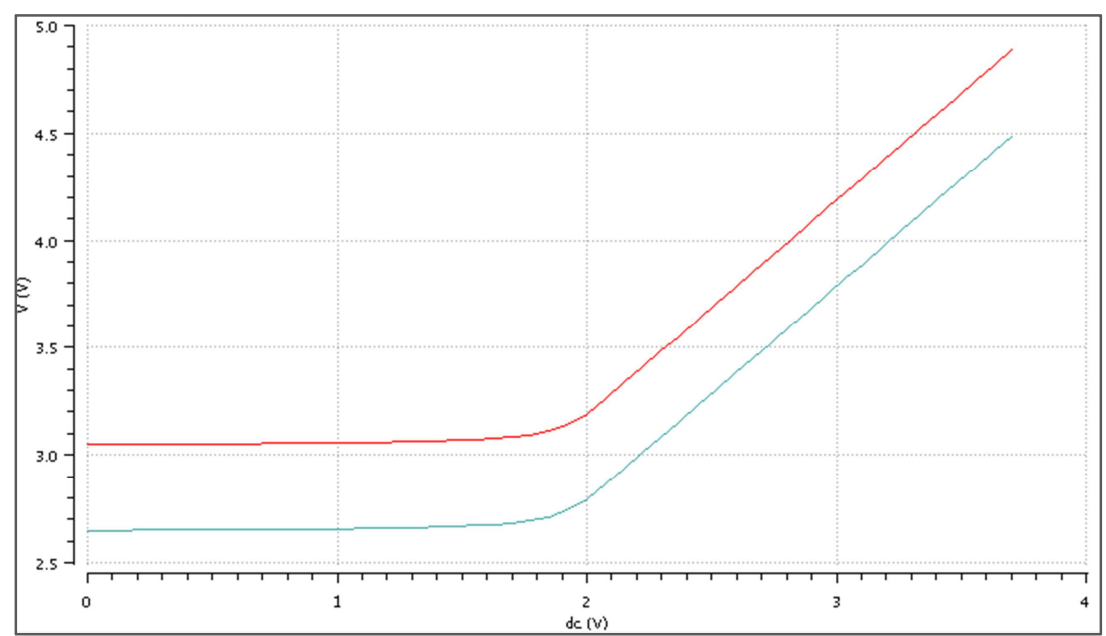

Figure 7. $V_{\text {drain }}($ bottom curve) and Vsource (top curve) of the PMOS-ISFET versus the Vgate sweep (x axis).

\section{Discussion}

\subsection{Individual Components Simulation}

For the current source, from Fig. 5, positive current is 19.95 $\mu \mathrm{A}$ and the negative current is $-19.95 \mu \mathrm{A}$. The current source circuit design is similar but with two output current terminals switched in positions. As 5 percent error current sources are possible with transistor beta value mismatch issues. The error of $0.05 / 20=2.5$ percent is acceptable. In fact, the contract specification of AMS (Austria Microsystem) technology laboratory standards is 5 percent error range due to the imperfections of mismatching of the transistors dimensions. The simulation error of 2.5 percent is actually within acceptable in emulation of manufacturing imperfections in labs.
From Fig. 6, at $\mathrm{V}_{\text {gate }}=1.6128$ volts, the current is $248 \mu \mathrm{A}$ while the voltage across the transistor is $5.0 \mathrm{~V}$. The resistance equivalent at this point is $5.0 \mathrm{~V} / 248 \mu \mathrm{A}=20.16 \mathrm{k}$, which is transistor-resistor within 2 percent deviation from the $20 \mathrm{k}$ resistor value. As five percent error resistors are still used in analog lab environment, the simulated resistor by NMOS transistor is acceptable.

\subsection{Sensor Circuit Simulation}

In the whole process Vsource-Vdrain $=0.4$ Volts, which is the $20 \mathrm{k} * 20 \mu \mathrm{A}$ difference across the resistor. The resistor circuit's top and bottom nodes are connected with the operational amplifiers of negative feedback as seen in Fig. 7. The voltages across the PMOS-ISFET is thus following the voltages across the resistor as the PMOS-ISFET's nodes are 
connected on the other end of the operational amplifier.

As seen in (1), for a fixed $I_{\text {drain }}$ across the PMOS-ISFET as the operational amplifiers turned on, the $V_{G S}-\left|V_{T}\right|$ needs to be constant as $V_{D S}$ is constant at $0.4 \mathrm{~V}$. Thus as the Vgate increases as a result of the $\mathrm{pH}$ increasing in the solution, the Vs increments has to follow the $\mathrm{Vg}$ increments in a 1:1 ratio. With a constant $V_{d s}, V_{d}$ will need to increase linearly with a gradient of one as well.

The gradient $=1$ increment of the Vdrain and Vsource is a result of the (1).

$$
\mathrm{Id}=\mu \operatorname{Cox} \frac{\mathrm{W}}{\mathrm{L}}\left(\left(V_{G S}-\left|V_{T}\right|\right) V_{D S}-\left(\frac{V_{D S}}{2}\right)^{2}\right)\left(1+\lambda V_{D S}\right)
$$

Note that (1) specifies that drain current $I_{d}$ varies linearly in $1: 1$ ratio as the drain-source voltage $\mathrm{V}_{\mathrm{DS}}$ changes in the linear regime ISFET operation.

The Glasgow Cumming's research group adapted the sensitivity of the ISFET at $46 \mathrm{mV} / \mathrm{pH}$ [6], while the theoretical maximum sensitivity of the ISFET measured by Bergveld at $59.2 \mathrm{mV} / \mathrm{pH}$ division [7]. We thus proposed a sensitivity of 50 $\mathrm{mV} / \mathrm{pH}$. With the CMOS compatible technology at the Scottish Microelectronics Centre and our partner Austria Microsystems, we can achieve this sensitivity level in chip manufacture.

In clinical research cancer cells function in acidic environment about $0.4 \mathrm{pH}$ divisions lower than the normal cells and normal cell's $\mathrm{pH}$ 7.2-7.6. With a lower bound of cancer cells typically not lower than $\mathrm{pH}$ of 5 . We are interested in $\mathrm{pH}$ range $\mathrm{pH} 4-8$, with a bit of safety margin in cancer cell monitoring. Thus we designed the device to record $4 \mathrm{pH}$ units difference. $4 \mathrm{pH} \times 50 \mathrm{mv} / \mathrm{pH}=200 \mathrm{mV}$.

To achieve this $200 \mathrm{mV}$ Vgate sensitivity range, we can pick a linear region on the Vsource graph from 3.5 volts to 3.7 volts, as Vgate and Vsource changes at this region is linear with gradient one. The Vgate corresponding to the Vsource value of 3.5 volts is 2.29 volts. The span of 2.29 volts to 2.49 volts is thus the Vgate variance region for the $\mathrm{pH}$ changes of the tissues being monitored by the ISFET array.

Clinical studies illustrates that the $\mathrm{pH}$ level of cancer cell microenvironment is typically lower than normal cells of the same patient by 0.2 to 0.6 units with an average of 0.4 units [8].

Thus we can conclude that our simulation designed ISFET sensor that has decent sensitivity to monitor cancer growth of post-surgery prognosis. The simulation result of the ISFET electrochemical sensors showed that the array sensor can convert $\mathrm{pH}$ information of tissues into voltage outputs. With a delicate ISFET device in the actual lab that has sensitivity to a few or even one $\mathrm{mV}$ in measurements, we can measure changes of the organ after surgery up to:

$$
1 \mathrm{mV} / 50(\mathrm{mV} / \mathrm{pH})=0.02 \mathrm{pH}
$$

This minute-to-minute measurement of $0.02 \mathrm{pH}$ divisions should definitely be capable of delivering prognosis report of any potential alarms in cancer re-growth of the patient's organ as a typical $0.4 \mathrm{pH}$ is dropped from normal to tumour cell mutation.

$$
0.4 \mathrm{pH} / 0.02 \mathrm{pH}=20 \text { steps. }
$$

Thus after the digital readout circuitry of the analogue ISFET sensor array output, the designer can provide a chart of 20 steps of severity level of a possible mutation of cancer for the organ or tissue being monitored after the surgery.

Similar calculations for biasing range sensitivity can be conducted for O2-FET sensors. O2-FET can be viewed as a modified ISFET sensor that measures oxygen level indirectly by measurements of $\mathrm{pH}$. The exact mechanism of oxygen level concentration estimation is via measurement of $\mathrm{pH}$ change in the process of oxygen and proton's combination reaction into water. One molecule oxygen taking four hydrogen ions and four electrons in producing of two molecules of $\mathrm{H}_{2} \mathrm{O}$ as illustrated in (3).

$$
\mathrm{O}_{2}+4 \mathrm{H}^{+}+4 \mathrm{e}^{-} \rightarrow 2 \mathrm{H}_{2} \mathrm{O}
$$

Equation (3) illustrates the chemical reactions mechanisms of O2-FET sensors. As the electrons are ejected into the solution, which are connected to the gate electrode, the $\mathrm{V}_{\mathrm{GS}}$ of (1) thus varies and the oxygen level is henceforth monitored by the $\mathrm{O}_{2}$ FET a similar mechanism as the $\mathrm{pH}$ level by the ISFET.

In the transducer's conversion calculations of O2-FET, similar to our simulation for the biasing scheme for ISFET, the $\mathrm{V}_{\text {gate }}$ is changing as the oxygen concentration changes. If a similar manufacture procedure is applied for the O2-FET, the oxygen sensor simulation result should resemble that a linear response of input $\mathrm{V}_{\text {gate }}$ voltage to the output $\mathrm{V}_{\text {drain }}$ with a specific optimal biasing voltage range.

Research has shown most cancer growth and metastasis occur in metastatic environments of low oxygen concentration and tumour hypoxia is an important indicator of cancer prognosis. Low oxygen levels in cancer development are denoted as hypoxia [9].

Our simulation work paved the path for future research. The possible future research that can be taken by the SMC team on the extension research of the implantable Microsystems for personalized anti-cancer therapy.

For example, one may consider the employment of a digital microprocessor that is programmable in controlling which signal to read over the minute-to-minute monitoring of the device.

Another area of future research is the variety of chemical signals to be measured. Aside from the oxygen FET circuit that can also be implemented in the instrumentation that is similar to the ISFET instrumentation we simulated. As researchers discovered that silicon nanowire based nanostructure field-effect transistors are compatible with conventional Si-based technology [10]. Scientists already combined the FET sensors with immobilized anti-human serum albumin (a type of globular protein), polyvinylbutyral membrane to make immuno-FET [11]. Future research may also consider employing similar mechanisms in examining cancer-related proteins such as Oct-4 (octamer-binding transcription factor 4). This year's research advances in electrochemical sensing includes the application of 
nano-structured gold double-stranded (ds) DNA sensor that can bind to beta protein 1 for breast cancer detections [12]. Similar mechanism may be explored for incorporation of a binding site for Oct-4 cancer marker protein in dsDNA electrochemical sensors.

The final implantable product device needs to be biocompatible prior to the implant test in patients. It is important that the implanted device will not induce side-effects due to lost packaging materials inside human organ or being toxic to the tissues. A proposed solution is to incorporate a layer of parylene encapsulation on top of the MEMS device before the implant. The parylene is an American Food and Drug Administration (FDA) approved material for implantable devices and can be incorporated as encapsulation layer via the deposition process of the MEMS wafer chip.

Additional research may extend in the direction of making the sensor smaller, and more sensitive via nano-technology. Various methods of binding proteins into nano-structured sensors have already been explored [12]. Future research of the SMC may also be involved in the field of integrating these protein-binding carbon nanotubes into electrochemical probes. This research may help reducing the size of the electrochemical sensor and hence making the probes more miniaturized for various extracellular micro-environments in cancer monitoring.

\section{Conclusion}

Our simulation result demonstrated that appropriate biasing of ISFET $\mathrm{V}_{\text {gate }}$ voltage is within 2.29-2.49 volts, in the case of $\mathrm{pH}$ measurements. The $\mathrm{pH}$ readout sensitivity can be up to $0.02 \mathrm{pH}$ in a minute-to-minute $\mathrm{pH}$ probing device for cancer prognosis tracking. Other chemical probes such as O2-FET and Oct4 sensors may also be included for synthetic cancer diagnostics. Similar conversion equations of the input and output for the electrochemical sensors and digitization of the output interpretations need to be formulated for the complete device design. Our results prepared us for future research in multifunctional electrochemical sensor array systems of different chemical sensor types for post-surgery cancer recurrence monitoring.

\section{Acknowledgments}

The author thank EPRSC grant for supporting project IMPACT (Implantable Microsystems for Personalized Anti-Cancer Therapy) 5.2 million pounds EPSRC awarded to the SMC (Scottish Microelectronics Centre) and School of Engineering of Edinburgh University. Its contents are solely the responsibility of the author and do not necessarily represent the official views of the EPSRC.

\section{References}

[1] DeSantis, C. et al., "Cancer Treatment and Survivorship Statistics", 2014, CA: A Cancer Journal for Clinicians, vol. 64 (4), pp. 252-271.

[2] Cumming, D., "ISFET sensor system for real-time detection of extracellular $\mathrm{pH}$ oscillations in slime mould". Electronics Letters, 2012, 48, pp. 144-146.

[3] Corbin, E. et al. "Biophysical properties of human breast cancer cells measured using silicon MEMS resonators and atomic force microscopy", lab on a chip, 2015, vol. 15. pp. 839-847.

[4] Chen, P., Humayun, M., Tai, Y., "Implantable parylene-based wireless intraocular pressure sensor”. IEEE 21st international conference on Micro Electro Mechanical Systems, 2008: 5861.

[5] Bergveld, P., "Thirty years of ISFETOLOGY What happened in the past 30 years and what may happen in the next 30 years." Sensors and Actuators B, 2003, 88: 1-20.

[6] Milgrewa, M., Riehleb, M., Cumming, D. "A large transistor-based sensor array chip for direct extracellular imaging." Sensors and Actuators B, 2005, 111: 347-353.

[7] Hal, R., Eijkel, J., Bergveld, P. A novel description of ISFET sensitivity with the buffer capacity and double-layer capacitance as key parameters. Sensors and Actuators B, 24-25, 1995, pp. 201-205.

[8] Gerweck, L., Seetharaman, K. cellular $\mathrm{pH}$ gradient in tumor versus normal tissue: potential exploration for the treatment of cancer. Cancer Research. 1996, 56 (6):1194-1198.

[9] Wanekaya, A. et al. "Nanowire-Based Electrochemical Biosensors. Electroanalysis" 2006, vol. 18 (6), pp. 533-550.

[10] Wykoff, C., Beasley, N. et al. Hypoxia-inducible Expression of Tumor-associated Carbonic Anhydrases. Cancer Research. 2000, 60, pp. 7075-7083.

[11] Atlasi, Y., Mowla, S. et al: 'OCT-4, an embryonic stem cell marker, is highly expressed in bladder cancer', International Journal of Cancer, 2007, 120, (7), pp. 1598-1602.

[12] Peres, W. et al. "Nanostructured gold dsDNA sensor for early detection of breast cancer by beta protein 1 (BP1)", Journal of electroanalytical chemistry, 2015; vol. 751 pp. 49-56. 